\title{
Breast cancer-specific TRAIL expression mediated by miRNA response elements of let-7 and $m i R-122$
}

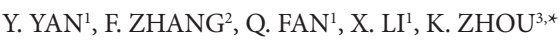 \\ ${ }^{1}$ Department of Oncology, The First Affiliated Hospital of Zhengzhou University, Zhengzhou 450052, China; ${ }^{2}$ Department of Gastroenterology, \\ The First Affiliated Hospital of Zhengzhou University, Zhengzhou, China; ${ }^{3}$ Department of Thoracic Surgery, The First Affiliated Hospital of \\ Zhengzhou University, Zhengzhou, China
}

*Correspondence to: kunzhouhn@gmail.com

Received December 10, 2013 / Accepted March 24, 2014

\begin{abstract}
Breast cancer is a highly aggressive malignancy and always has a poor prognosis. The current therapeutic strategies including surgery, chemotherapy and radiotherapy benefit little for patients survival. Although tumor necrosis factor-related apoptosis-inducing ligand (TRAIL) can induce apoptosis in a wide range of cancer cells, such as breast cancer, it also has cytotoxicity to normal cells. To improve the selectivity of TRAIL expression to breast cancer cells, we inserted miRNA response elements (MREs) of let-7 and miR-122 into a TRAIL-expressing adenoviral vector (Ad-TRAIL-MRE-7-122) to restrict its expression within breast cancer cells. qPCR assay confirmed that the levels of let-7 and miR-122 were downregulated in breast cancer samples and cell lines, compared with normal tissues and cell lines. Luciferase assay indicated that MREs of let-7 and miR-122 was able to confer luciferase expression with the selectivity to breast cancer. Ad-TRAIL-MRE-7-122 highly expressed TRAIL in breast cancer cells, but not in normal cells. The breast cancer-specific apoptosis was detected after the infection of Ad-TRAIL-MRE-7-122. The viability of breast cancer cells, rather than normal cells, was reduced by the treatment of Ad-TRAIL-MRE-7-122. Animal experiments further confirmed that Ad-TRAIL-MRE-7-122 and Ad-TRAIL both greatly impeded the growth of breast cancer xenograft in mice. Taken together, we constructed a TRAIL-expressing recombinant adenovirus regulated by MREs of let-7 and $m i R-122$ and showed evidences that this strategy may be promising for breast cancer treatment.
\end{abstract}

Key words: breast cancer, adenovirus, let-7, miR-122, TRAIL

Breast cancer is the most common type of cancer in women with high aggressiveness [1]. However, the current therapeutic modalities, such as surgery, chemotherapy and radiotherapy, benefit little for the survival of the patients. Thus, novel strategies were highly required for efficient breast cancer treatment.

Gene therapy, a strategy that expresses exogenous tumorsuppressor genes within cancer cells, has been studied for decades. Among various biological vectors, adenovirus is widely used for gene therapy because of its high volume and titer [2]. Researchers have developed some adenoviral vectors to introduce exogenous genes into breast cancers [3, 4]. Many tumor suppressor genes have been used for breast cancer treatment, consisting mainly of cell-autonomous genes. However, the therapeutic effect of these genes is quite limited because their action is only restricted only within the cells infected by adenoviruses. Therefore, more potent anti-tumor genes should be used for breast cancer therapy, such as cytokines.

Tumor necrosis factor-related apoptosis-inducing ligand (TRAIL) is well documented to exert high anti-tumor activity to a wide range of cancers, including breast cancer [5]. TRAIL can recognize and bind the corresponding receptors located in cell membranes, TRAILR1 and TRAILR2, to activate downstream proapoptotic proteins, caspase 8 and 10. In turn, the executors of apoptotic pathway, caspase 3, were activated to degrade its substrates [6]. However, TRAIL is also able to induce apoptosis in liver cells [7]. The unbiased infection of adenoviral vector to all kinds of cells probably leads to hepatotoxicity. Although some groups used TERT promoter to prevent TRAIL expression from normal cells, the relatively low activity of TERT promoter compromised the expression level of the inserted therapeutic genes [8]. 
miRNAs are a class of non-encoding RNAs consisting of 20-22 nucleotides. Mature miRNAs are generated from long precursor RNAs through Dicer- and Drosha-mediated processing complexes. miRNA can recognize and bind the miRNA response elements (MREs) sequences in the 3'UTR of target mRNAs, degrade these mRNA molecules or inhibit their translation [9]. It has been well documented that many miRNAs are differentially expressed between malignant and normal cells, including breast cancer [10]. Theoretically, low levels of certain miRNAs enables delivered genes selectively expressed within cells when their MREs are used for regulation. More importantly, the expression of inserted genes can be suppressed in normal cells because of the overexpression of selected miRNAs. However, no publication that focuses on the MRE-regulated gene therapy for breast cancer is available, although this strategy has been tested for some other types of cancer [11-14].

In this study, we used MREs let-7 and miR-122 to confer TRAIL expression with high selectivity to breast cancer. Experimental evidences showed that this strategy is feasible, efficient and biosafe.

\section{Materials and methods}

Cell line cultures. Human breast cancer cell lines, MCF-7 and MDA-MB-231, and human normal lung fibroblast cell line MRC-5 were purchased from American Type Culture Collection (Manassas, VA). Human breast cancer cell line, Bcap37, and Human normal liver cell line, L-02, were obtained from Shanghai Cell Collection (Shanghai, China). Cells were cultured using recommended media supplemented with $10 \%$ of fetal bovine serum (FBS), $4 \mathrm{mM}$ glutamine, 100 units $/ \mathrm{ml}$ penicillin, and $100 \mu \mathrm{g} / \mathrm{ml}$ streptomycin in a $5 \% \mathrm{CO}_{2}$ and humidified atmosphere at $37^{\circ} \mathrm{C}$.

Quantitative PCR (qPCR). For miRNA detection in breast cancer specimen, fresh cancerous and noncancerous breast tissues were obtained with written informed consent from all patients according to protocols approved by Ethical Review Board in The First Affiliated Hospital of Zhengzhou University (Zhengzhou, China). All patients underwent surgical resection of primary breast cancer at Department of Thoracic Surgery, The First Affiliated Hospital of Zhengzhou University (Zhengzhou, China).

Total RNA was extracted from these specimens with Trizol solution (Sigma-Aldrich, MO). Reverse transcription reaction was performed with TaqMan ${ }^{\oplus}$ MicroRNA Reverse Transcription Kit (Applied Biosystems) following the manufacturer's instructions. qPCR was performed using TaqMan ${ }^{\circledR} 2 \times$ Uni- $^{-}$ versal PCR Master Mix (Applied Biosystems) on CFX96 ${ }^{\text {mx }}$ Real-Time PCR Detection System (Bio-Rad Laboratories, CA) supplied with analytical software.

To determine TRAIL mRNA level in adenovirus-infected cells, 10 MOI of indicated adenoviruses were added to cell cultures. After $48 \mathrm{~h}$, cells were harvested for RNA extraction, followed by being transcribed into cDNAs using Rever Tra
Ace qPCR RT Kit (Toyobo, Japan) according to the manufacturer's instructions. qPCR was performed using TaqMan ${ }^{\circledR} 2 \times$ Universal PCR Master Mix (Applied Biosystems) on CFX96 $6^{\text {tw }}$ Real-Time PCR Detection System (Bio-Rad Laboratories, CA) supplied with analytical software. TRAIL-forward primer: 5'-gacctgcgtgctgatc-3'; TRAIL-reverse primer: 5'-taaaagaagatgacag -3 '.

Luciferase reporter construction and assay. A DNA fragment containing 2 copies of MREs of let-7 and miR-122 (5'-GCCCTCGAGACAAACACCTCTTCCAACAAACACCTCTTCCAACAAACACCACACTCCACAAACACCACACTCCGCGGCCGCGGC-3') were synthesized, annealed and inserted into the $\mathrm{XhoI}$ and NotI sites of psiCheck2 vectors (Promega, WI) immediately following the Renilla luciferase gene to construct MRE-regulated luciferase reporter, which were designated as psiCheck2-MRE-7-122.

After transfection of MCF-7, Bcap37, MRC-5 and L-02 cells and subsequent $48 \mathrm{~h}$ incubation, cells were harvested and treated with lysis buffer. Firefly and Renilla luciferase activities were determined with the Dual-Luciferase reporter system (Promega, WI) following the manufacturers protocol.

Adenovirus construction. Adenoviruses, Ad-EGFP and Ad-TRAIL, were kindly provided by Dr. Zhao (General Hospital of Chengdu Military Area Command of Chinese PLA, Chengdu, China). We constructed AdTRAIL-MRE-7-122 mainly according to the procedures described as follows. A DNA fragment containing two copies of MREs of let-7 and miR-122 (5'-GCCGATATCACAAАCACСТCTTCCAACAAACAССТCTTCCAAC AAACACCACACTCCACAAACACCACACTCCGATATCGGC-3') were released by digested by EcoRV, followed by its insertion into the same site on pShuttle-CMV-TRAIL (also gifted by Dr. Zhao) to generate pShuttle-CMV-TRAILMRE-7-122. pShuttle-CMV-TRAIL-MRE-7-122 was then cotransfected with pAdEasy into HEK-293 cells. After plague purification for three times and PCR-based identification, the adenoviruses were harvested and then purified with the $\mathrm{CsCl}$ gradient centrifugation. The constructed adenovirus was designated as Ad-TRAIL- MRE-7-122. The titers of the involved adenoviruses were quantified with $\mathrm{TCID}_{50}$ method on HEK-293 cells and shown as plaque-forming units per milliliter ( $\mathrm{pfu} / \mathrm{ml})$. The structures of these adenoviruses were illustrated in Fig. 2A.

Immunoblotting assay. Total proteins were harvested with M-PER Mammalian Protein Extraction Reagent (Thermo Scientific, IL), separated using polyacrylamide gel electrophoresis and transferred onto $0.45 \mu \mathrm{m}$ nitrocellulose membranes. After being blocked with $5 \%$ fat-free dry milk for $2 \mathrm{~h}$. The membrane was incubated with primary antibody. The membrane was incubated overnight with the corresponding secondary antibody and visualized with SuperSignal West Dura Extended Duration Substrate (Thermo Scientific, IL). The involved antibodies were as follows. TRAIL (Santa Cruz), cleaved caspase-3 (Cell Signaling Technology), cleaved PARP (Cell Signaling Technology), GAPDH (Santa Cruz). 
A

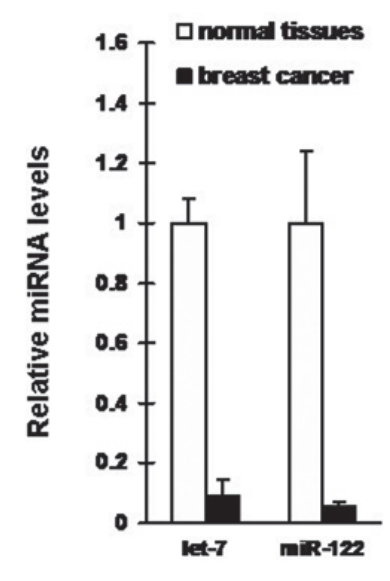

B

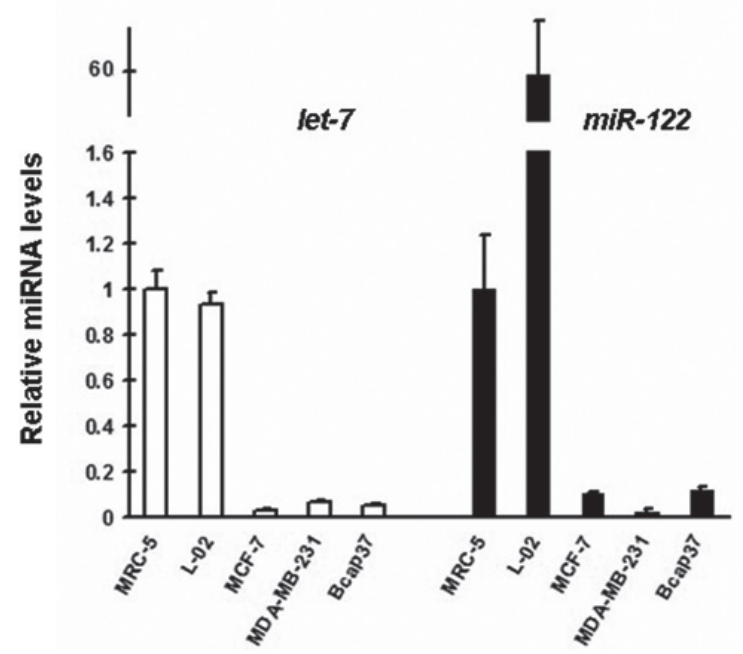

Figure 1. let-7 and $m i R-122$ were downregulated in breast cancers. qPCR assays were used to examine the expression of let-7 and $m i R-122$ in breast cancer and normal tissues $(n=10)(A)$ as well as breast cancer cell lines and normal cell lines $(B)$. The bars represented means \pm SD of three independent experiments.

Detection of TRAIL by ELISA. 10 MOI of indicated adenoviruses were added to cell media. After $48 \mathrm{~h}$, the two-antibody sandwich ELISA was used to detect human TRAIL expression in the supernatant of cell cultures. The used antibodies are monoclonal mouse anti-human TRAIL antibody (R\&D Systems), peroxidase-conjugated rabbit antigoat IgG (H\&L) and goat anti-human TRAIL antibody (R\&D Systems). The absorbance was read at a $450 \mathrm{~nm}$ wavelength. A standard curve was used to determine the concentration of TRAIL.

Cell viability assay. Adenoviral vectors of indicated MOIs were added to cell cultures for 6 days. $50 \mu$ of 3-(4,5-dimethylthiazol-2-yl)-2,5-diphenyltetrazolium bromide (MTT) (1 mg/ml) was added. $4 \mathrm{~h}$ later, MTT was removed and $150 \mu \mathrm{l}$ of dimethyl sulfoxide (DMSO) was added. The spectrophotometric absorbance was measured on a model 550 microplate reader (Bio-Rad Laboratories, Hercules, CA) at $570 \mathrm{~nm}$ with a reference wavelength of $655 \mathrm{~nm}$. Cell viability was calculated according to the following formula: Cell viability = absorbance value of infected cells / absorbance value of uninfected control cells.

Flow cytometrical analysis of apoptotic rates. The cells were infected with Ad-EGFP, Ad-TRAIL or Ad-TRAILMRE-7-122 of 10 MOI. 48 h later, cells were fixed with $70 \%$ cold ethanol and stained with Annexin V-FITC/PI Apoptosis Detection Kit (Biovision, CA) following the manufacturer's instructions. The percentage of apoptotic cells was analyzed by flow cytometry.

Animal experiments. Procedures for animal experiments were all approved by the Committee on the Use and Care on Animals in The First Affiliated Hospital of Zhengzhou University (Zhengzhou, China).

Bcap37 tumor xenograft was established by injecting $2 \times 10^{6}$ cells at the right flanks of 5 -week-old male $\mathrm{BALB} / \mathrm{c}$ nude mice. As soon as tumors grew to $6-8 \mathrm{~mm}$ in diameter, 24 mice were randomly divided into 4 groups $(n=6)$. The mice were intratumorally injected with PBS, $2 \times 10^{8}$ pfu of Ad-EGFP, $2 \times 10^{8}$ Ad-TRAIL or $2 \times 10^{8}$ Ad-TRAIL-MRE-7-122. The injections were repeated every other day for five times with a total dosage of $1 \times 10^{9} \mathrm{pfu}$ of adenoviruses. Tumor diameter was measured by periodic measurements with calipers and volume was calculated using the following formula: tumor volume $\left(\mathrm{mm}^{3}\right)=$ maximal length $(\mathrm{mm}) \times$ perpendicular width $(\mathrm{mm})^{2} / 2$.

\section{Results}

The abundance of $l e t-7$ and $m i R-122$ was downregulated in breast cancer cells. First of all, we investigated the expression levels of let-7 and miR-122 in breast cancer. In 10 breast cancer specimens from patients, the levels of let-7 and $m i R-122$ were found to be reduced in comparison with normal tissues (Fig. 1A). In addition, we also assessed the expression of these two miRNAs in several cell lines. The results indicated that the levels of let-7 and $m i R-122$ were both reduced in breast cancer cell lines, compared with normal cell lines (Fig. 1B).

MREs of let-7 and $m i R-122$ ensured the expression of exogenous gene to be selectively expressed in breast cancer cells. We constructed a luciferase reporter vector under the regulation of MREs of let-7 and $m i R-122$, in order to determine if the existence of MREs of let-7 and $m i R-122$ can regulate the expression of inserted genes, (Fig. 2A). The data showed that combination of MREs of let-7 and miR-122 suppressed luciferase activity in normal cell lines, MRC-5 and L-02. In contrast, luciferase expression was slightly affected by the action of MREs of let-7 and miR-122 in MCF-7 and Bcap37 breast cancer cells (Fig. 2B). 
A

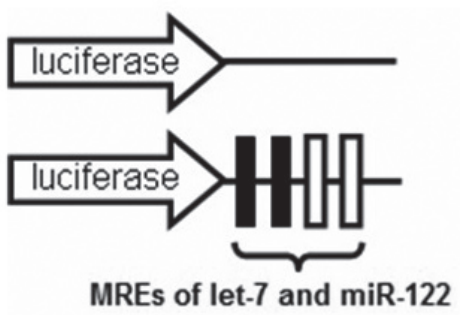

B

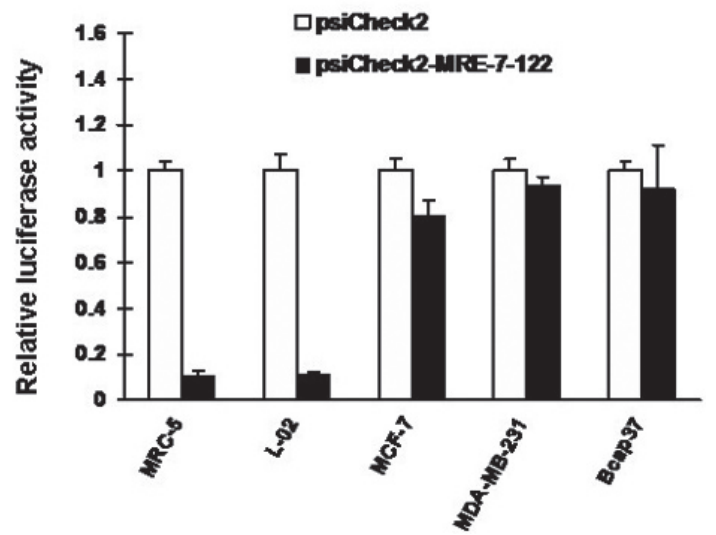

Figure 2. miRNA response elements of $l e t-7$ and $m i R-122$ regulated breast cancer-selective TRAIL expression. (A) luciferase reporter vectors were schemed here. (B) The indicated cell lines were transfected with psiCheck2 or psiCheck2-MRE-7-122, followed by measuring luciferase expression. The bars represented means \pm SD of three independent experiments.

Application of MREs of let-7 and miR-122 conferred TRAIL expression with high selectivity to breast cancer. Subsequently, we constructed a recombinant adenovirus expressing TRAIL under the regulation of MREs of let-7 and miR-122 by inserting 2 copies of these elements immediately following TRAIL-encoding region, namely Ad-TRAIL-MRE7-122 (Fig. 3A). The immunoblot, qPCR and ELISA assays all showed that Ad-TRAIL-MRE-7-122 had comparative level of TRAIL with Ad-TRAIL in the tested breast cancer cells (Fig. 3B-3D). However, the expression level of TRAIL mediated by
A

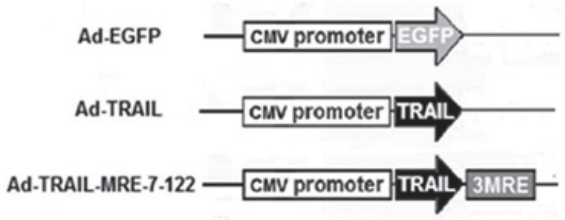

C

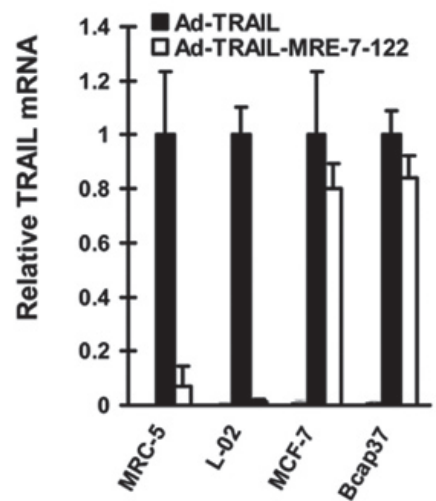

B

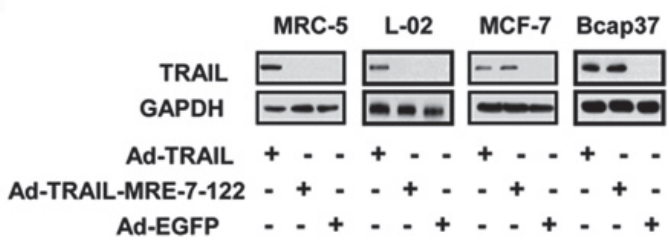

D

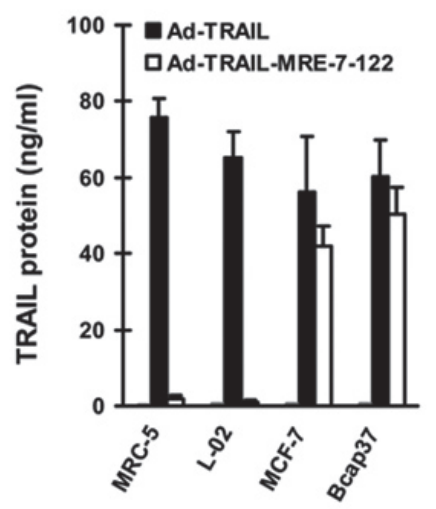

Figure 3. TRAIL was selectively expressed by adenoviral vectors under MREs of let-7 and $m i R-122$. (A) The structures of involved adenoviral vectors were schemed here. The indicated cell lines were infected with different adenoviruses of $10 \mathrm{MOI} .48 \mathrm{~h}$ later, the expression of TRAIL was detected by immunoblot (B), qPCR (C) and ELISA (D) assays. The bars represented means \pm SD of three independent experiments. 
A

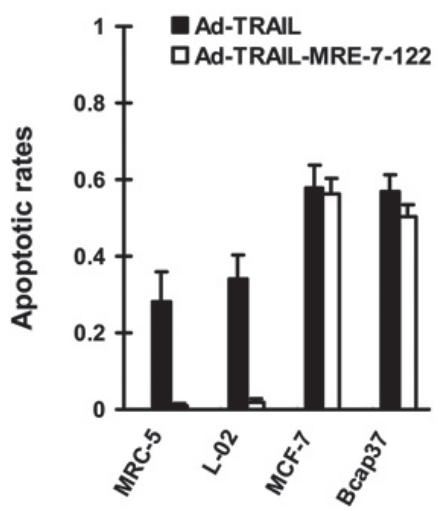

B

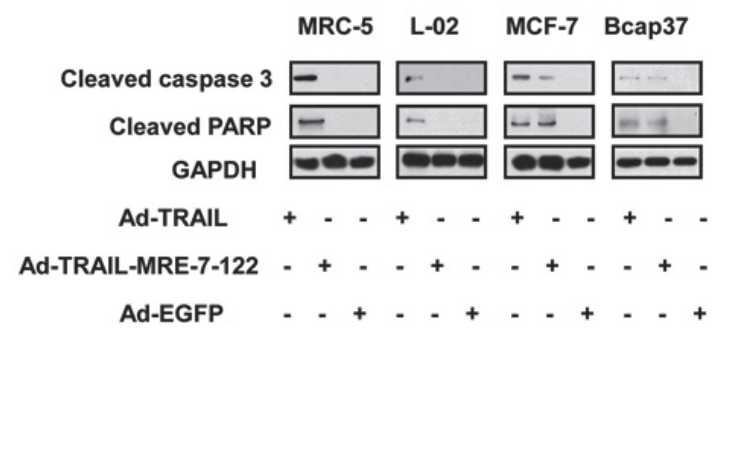

Figure 4. Ad-TRAIL-MRE-7-122 induced apoptosis specifically in breast cancer cells. (A) MRC-5, L-02, MCF-7 and Bcap37 cell lines were infected with different adenoviruses of $10 \mathrm{MOI}$. Then, apoptotic rates were assessed using flow cytometrical analysis of Annexin V/PI expression. The bars represented means \pm SD of three independent experiments. (B) Immunoblotting assay was performed to detect the expression of cleaved caspase 3 and PARP with GAPDH as loading control.

Ad-TRAIL-MRE-7-122 is much lower in normal cell lines, MRC-5 and L-02, than Ad-TRAIL (Fig. 3B-3D).

Ad-TRAIL-MRE-7-122 induced specific activation of apoptotic pathway in breast cancers. Given that TRAIL can activate apoptotic pathway in a wide range of cells, we next determined the apoptotic rates of breast cancer and normal cells infected with Ad-EGFP, Ad-TRAIL and Ad-TRAIL-MRE-7122 by flow cytometry. Increased percentages of apoptotic cells was detected in breast cancer cells infected with Ad-TRAILMRE-7-122 or Ad-TRAIL as well as normal cells transduced with Ad-TRAIL. In contrast, there was no significant apoptosis in MRC-5 and L-02 cells infected with Ad-TRAIL-MRE-7-122 (Fig. 4A). Further Immunoblot analysis of cleaved caspase 3 and PARP also revealed that apoptotic pathway was activated in breast cancer cells infected with Ad-TRAIL or Ad-TRAILMRE-7-122 as well as normal cells infected with Ad-TRAIL. However, the cleavage of these two apoptosis-related proteins was not detected in normal cells transduced with Ad-TRAILMRE-7-122 (Fig. 4B).

MRE-regulated TRAIL expression leads to selectivity cytotoxicity to breast cancer cells. MTT assay was employed to evaluate the survival of breast cancer and normal cells after the transduction of Ad-EGFP, Ad-TRAIL or Ad-TRAIL-MRE7-122. Ad-TRAIL-MRE-7-122 only had a slight cytotoxicity to MRC-5 and L-02 cells at high MOI (Fig. 5A and 5B). In contrast, Ad-TRAIL highly suppressed the survival of the two cell lines (Fig. 5A and 5B). Furthermore, the results revealed that Ad-TRAIL-MRE-7-122 greatly reduced the viability of breast cancer cells by a similar extent to Ad-TRAIL (Fig. 5C and 5D).

Ad-TRAIL-MRE-7-122 greatly reduced the tumorigenesis of breast cancer in vivo. Then, we wondered if Ad-TRAIL-MRE-7-122 can suppress the growth of breast cancer xenograft in vivo. Bcap37 cells were subcutaneously injected into the flanks of nude mice. According to the data about tumor volume, both of Ad-TRAIL-MRE-7-122 and Ad-TRAIL inhibited the growth of established tumors in mice and their effectiveness seemed to be similar (Fig. 6A and $6 \mathrm{~B})$.

We also investigated the expression of TRAIL protein in tumor section by immunohistological staining. The results indicated that Ad-TRAIL-MRE-7-122 and Ad-TRAIL both had a high level of TRAIL expression in vivo (Fig. 6C).

Ad-TRAIL-MRE-7-122 protected hepatic tissues from toxicity. Finally, we investigated if Ad-TRAIL-MRE-7-122 can prevent liver from TRAIL-induced toxicity. In another group of tumor-free mice, we observed that serum level of ALT was highly elevated in the mice injected with AdTRAIL. However, there was no significant difference in serum level of ALT between Ad-TRAIL-MRE-7-122- and Ad-EGFP or PBS-injected mice (Fig. 7A). Immunohistological staining further confirmed that TRAIL expression was detected in the liver section from Ad-TRAIL-treated mice, but not Ad-TRAIL-MRE-7-122 and Ad-EGFP (Fig. 7B).

\section{Discussion}

We firstly investigated miRNA expression profiles of breast cancer and corresponding noncancerous normal tissue. The data showed that let-7 and $m i R-122$ were significantly reduced in breast cancer, compared with normal tissue. The reduced expression of these two miRNAs allows their MREs to be used for regulate exogenous gene expression in a breast cancer-selective fashion. Luciferase reporter assay further demonstrated that MREs of let-7 and miR-122 was able to suppress luciferase ac- 
A

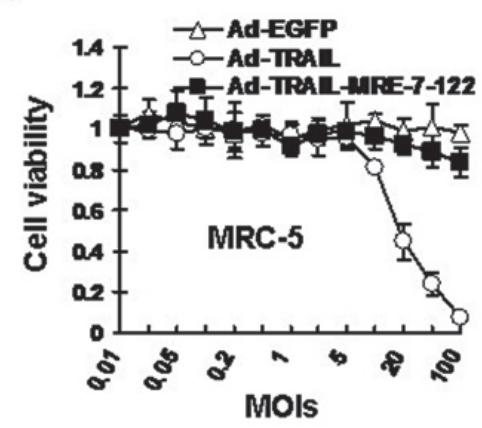

C

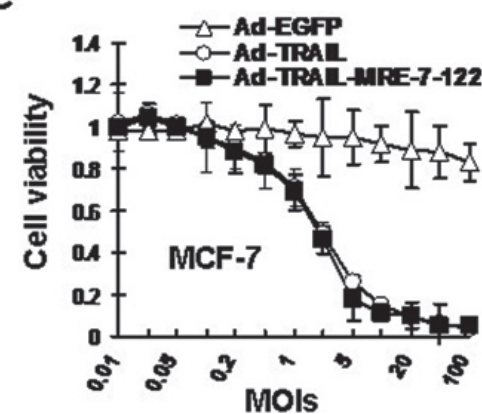

B

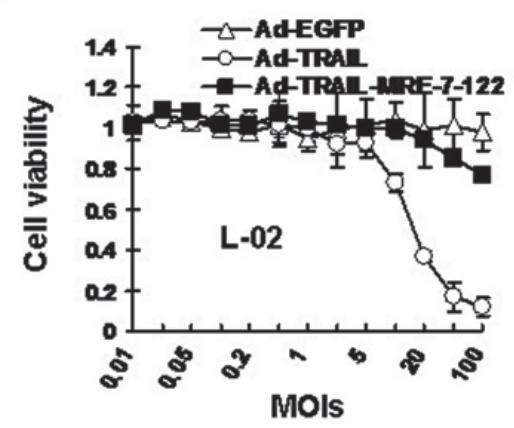

D

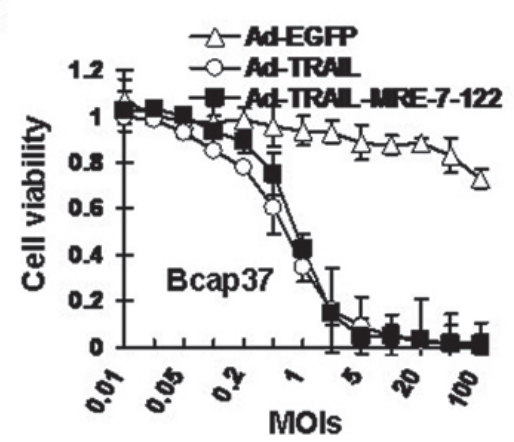

Figure 5. Ad-TRAIL-MRE-7-122 displayed selective cytotoxicity to breast cancer cells. After the infection of different adenoviruses, we used MTT assays to estimate the survival rates of breast cancer and normal cells. The curves represented means \pm SD of three independent experiments. (A) MRC-5. (B) L-02. (C) MCF-7. (D) Bcap37.

A

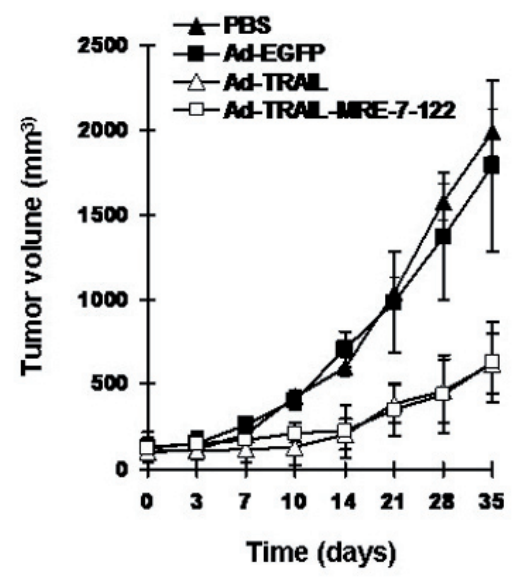

C

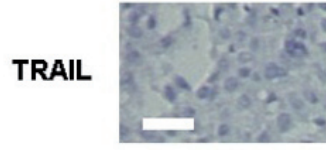

Ad-EGFP
B
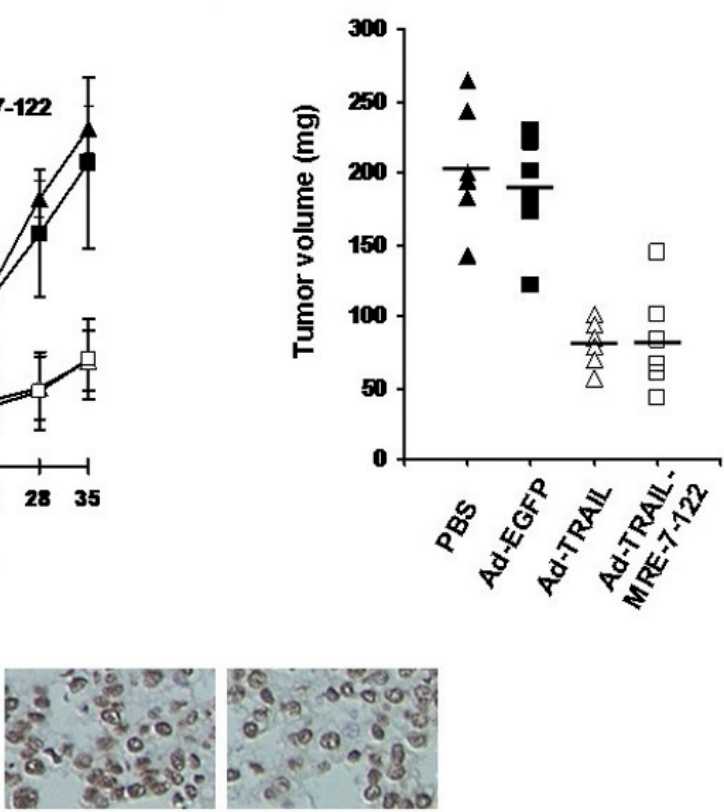

Ad-TRAIL

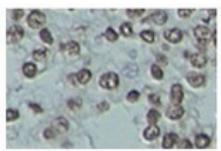

Ad-TRAILMRE-7-122

Figure 6. Ad-TRAIL-MRE-7-122 minimized the tumorigenecity of breast cancer in vivo. (A) The volumes of established breast cancer in mice were shown as means \pm SD, as well as their weight. (C) The expression of TRAIL was evaluated in the tumor sections derived from different groups of nude mice by immunohistological staining. The bar indicated $50 \mu \mathrm{m}$. 


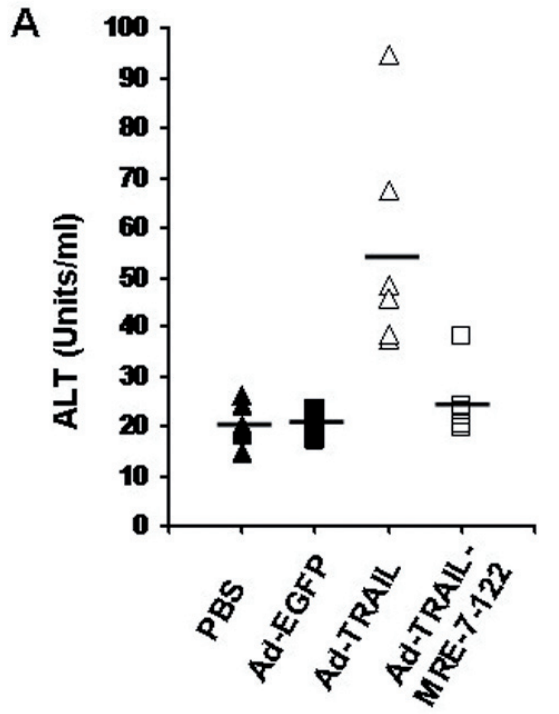

B

TRAIL

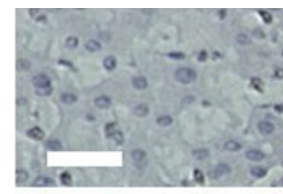

Ad-EGFP

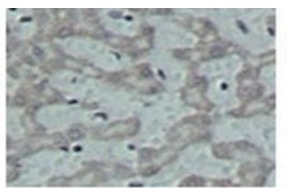

Ad-TRAIL

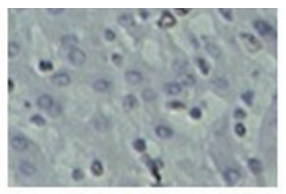

Ad-TRAILMRE-7-122

Figure 7. Ad-TRAIL-MRE-7-122 prevented hepatic tissues from TRAIL-induced toxicity. (A) Serum ALT levels were measured in the tumor-free mice after the intravenous injection of indicated adenoviral vectors. (B) The expression of TRAIL was evaluated in the liver section by immunohistological staining. The bar indicated $50 \mu \mathrm{m}$.

tivity in normal cells without significant reduction in luciferase expression in breast cancer cells. Subsequently, qPCR, immunoblot and ELISA assays revealed that TRAIL can be detected in all the cells infected with Ad-TRAIL. However, TRAIL expression mediated by Ad-TRAIL-MRE-7-122 displayed selectivity. MTT assays showed that Ad-TRAIL-MRE-7-122 and Ad-TRAIL both reduced the viability of the tested breast cancer cells. For normal cells, only Ad-TRAIL exerted an inhibitory effect on their survival, suggesting a biosafety to normal cells due to the existence of MREs of let-7 and miR122. Finally, in vivo data revealed that Ad-TRAIL-MRE-7-122 suppressed the growth of Bcap37 tumors in mice as potent as Ad-TRAIL and had no hepatotoxicity to the animals. In fact, the similar effects have been verified in some other types of cancers using MRE-based strategy, such as melanoma, glioma and bladder carcinoma [11-13].

The current strategy to improve the tropism of adenoviral vectors to breast cancers is using cancer-specific promoter to drive the expression of inserted genes, such as hTERT [5]. However, the capability of hTERT promoter to express exogenous gene is quite weak, thereby compromising the outcomes of gene therapy. In this study, we constructed a breast cancer-targeting adenoviral vector expressing TRAIL utilizing multiple MREs. The promoter we used to CMV promoter, which is among the most active promoters we have known. So the expression level of inserted genes is expected to much higher than that driven by tumor-specific promoter.

let-7 has been demonstrated to be downregulated in breast cancer cells [15]. The restoration of let-7 in breast cancer cells is able to suppress the tumorigenesis of breast cancer in vivo. $m i R-122$ is originally found to be enriched in liver tissues and suppress the progression of liver cancer by targeting multiple oncogenes [16]. Recently, $m i R-122$ was also indicated to be downregulated in breast cancer cells [17]. Therefore, MREs of the two miRNAs whose expressions were reduced in breast cancer cells are believed to be suitable for the regulation of TRAIL expression.

Taken together, we generated a TRAIL-expressing adenoviral vector under the regulation of MREs of let-7 and miR-122. This vector can restrict the expression of TRAIL within breast cancer cell without cytotoxicity to normal cells. This breast cancer-targeting adenovirus needs to be further improved for its clinical application.

\section{References}

[1] TINOCO G, WARSCH S, GLUCK S, AVANCHA K, MONTERO AJ Treating breast cancer in the 21st century: emerging biological therapies. J Cancer 2013; 4: 117-132. http://dx.doi. org/10.7150/jca.4925

[2] DOLOFF JC, WAXMAN DJ Adenoviral Vectors for Prodrug Activation-based Gene Therapy for Cancer. Anticancer Agents Med Chem 2013.

[3] ZHANG N, DONG X, SUN Y, CAI X, ZHENG C et al. Cytotoxic effects of adenovirus- and lentivirus-mediated expression of Drosophila melanogaster deoxyribonucleoside kinase on Bcap37 breast cancer cells. Oncol Rep 2013; 29: 960-966.

[4] HE X, LIU J, YANG C, SU C, ZHOU C et al. 5/35 fibermodified conditionally replicative adenovirus armed with p53 shows increased tumor-suppressing capacity to breast cancer cells. Hum Gene Ther 2011; 22: 283-292. http://dx.doi. org/10.1089/hum.2010.058

[5] LIN T, HUANG X, GU J, ZHANG L, ROTH JA et al. Longterm tumor-free survival from treatment with the GFP-TRAIL fusion gene expressed from the hTERT promoter in breast cancer cells. Oncogene 2002; 21: 8020-8028. http://dx.doi. org/10.1038/sj.onc. 1205926

[6] PAN G, O'ROURKE K, CHINNAIYAN AM, GENTZ R, EBNER R et al. The receptor for the cytotoxic ligand TRAIL. Science 1997; 276: 111-113. http://dx.doi.org/10.1126/ science.276.5309.111 
[7] CORAZZA N, JAKOB S, SCHAER C, FRESE S, KEOGH A et al. TRAIL receptor-mediated JNK activation and Bim phosphorylation critically regulate Fas-mediated liver damage and lethality. J Clin Invest 2006; 116: 2493-2499. http://dx.doi. org/10.1172/JCI27726

[8] HU Z, ROBBINS JS, PISTER A, ZAFAR MB, ZHANG ZW et al. A modified hTERT promoter-directed oncolytic adenovirus replication with concurrent inhibition of TGFbeta signaling for breast cancer therapy. Cancer Gene Ther 2010; 17: 235-243. http://dx.doi.org/10.1038/cgt.2009.72

[9] SONTHEIMER EJ, CARTHEW RW Silence from within: endogenous siRNAs and miRNAs. Cell 2005; 122: 9-12. http://dx.doi.org/10.1016/j.cell.2005.06.030

[10] TEKINER TA, BASAGA H Role of microRNA deregulation in breast cancer cell chemoresistance and stemness. Curr Med Chem 2013; 20: 3358-3369. http://dx.doi.org/10.2174/ 09298673113209990003

[11] LIU J, MA L, LI C, ZHANG Z, YANG G et al. Tumor-targeting TRAIL expression mediated by miRNA response elements suppressed growth of uveal melanoma cells. Mol Oncol 2013; 7: 1043-1055. http://dx.doi.org/10.1016/ j.molonc.2013.08.003

[12] BO Y, GUO G, YAO W MiRNA-mediated tumor specific delivery of TRAIL reduced glioma growth. J Neurooncol
2013; 112: 27-37. http://dx.doi.org/10.1007/s11060-012$\underline{1033-\mathrm{y}}$

[13] ZHAO Y, LI Y, WANG L, YANG H, WANG Q et al. microRNA response elements-regulated TRAIL expression shows specific survival-suppressing activity on bladder cancer. J Exp Clin Cancer Res 2013; 32: 10. http://dx.doi.org/10.1186/17569966-32-10

[14] ZHANG Z, ZHANG X, NEWMAN K, LIU X MicroRNA regulation of oncolytic adenovirus 6 for selective treatment of castration-resistant prostate cancer. Molecular cancer therapeutics 2012; 11:2410-2418. http://dx.doi.org/10.1158/ 1535-7163.MCT-12-0157

[15] YU F, YAO H, ZHU P, ZHANG X, PAN Q et al. let-7 regulates self renewal and tumorigenicity of breast cancer cells. Cell 2007; 131: 1109-1123. http://dx.doi.org/10.1016/ j.cell.2007.10.054

[16] MA L, LIU J, SHEN J, LIU L, WU J et al. Expression of miR122 mediated by adenoviral vector induces apoptosis and cell cycle arrest of cancer cells. Cancer Biol Ther 2010; 9: 554-561. http://dx.doi.org/10.4161/cbt.9.7.11267

[17] WANG B, WANG H, YANG Z MiR-122 inhibits cell proliferation and tumorigenesis of breast cancer by targeting IGF1R. PLoS One 2012; 7: e47053. http://dx.doi.org/10.1371/journal. pone. 0047053 